\title{
Tanshinone IIA induces growth inhibition and apoptosis in gastric cancer in vitro and in vivo
}

\author{
JIAN CHEN ${ }^{1}$, DONG-YUN SHI ${ }^{2}$, SHAN-LIN LIU ${ }^{2}$ and LIANG ZHONG ${ }^{1}$ \\ ${ }^{1}$ Department of Gastroenterology, Huashan Hospital, Fudan University; ${ }^{2}$ Biochemistry \\ Department of Shanghai Medical College, Fudan University, Shanghai, P.R. China
}

Received July 12, 2011; Accepted September 1, 2011

DOI: $10.3892 /$ or.2011.1524

\begin{abstract}
As a phytochemical derived from the roots of Salvia miltiorrhiza Bunge, Tanshinone IIA has been reported to possess anti-inflammatory and antioxidant activity. Studies in breast, colon, prostate and lung cancer indicate that Tanshinone IIA may exhibit a promising antitumor activity. However, systemic studies of the cytotoxic effects of Tanshinone IIA on gastric cancer have not been described. The present study offers a comprehensive evaluation of the antitumor effects of Tanshinone IIA in gastric cancer cells in vitro and in a mouse xenograft model. Cell viability and apoptosis in vitro were evaluated through the MTT assay and flow cytometry analysis. The results indicate that Tanshinone IIA can induce gastric cancer cell growth inhibition and apoptosis in a time- and concentration-dependent manner. Furthermore, we investigated the mechanism of the apoptotic effects induced by Tanshinone IIA. We found that Tanshinone IIA can not only cause cell cycle arrest in the $\mathrm{G} 2 / \mathrm{M}$ phase, but also trigger the intrinsic apoptotic signaling pathway. The results suggest that Tanshinone IIA may serve as an effective adjunctive reagent in the treatment of gastric cancer.
\end{abstract}

\section{Introduction}

As the second leading cause of cancer-related death, gastric cancer is a big threat to public health worldwide (1). Despite advances in the diagnosis and treatment of gastric cancer in the clinical setting within the last decades, the outcome of therapy remains disappointing (2). The conventional treatment approach of gastric cancer is gastrectomy combined with pre- or post-chemotherapy or radiotherapy (3). However, the side effects of chemotherapy or radiotherapy including multidrug resistance and cytotoxicity to normal cells restrict their

Correspondence to: Dr Liang Zhong, Department of Gastroenterology, Huashan Hospital, Fudan University, Shanghai 200040, P.R. China

E-mail: chen5120@yahoo.com

Key words: Tanshinone IIA, gastric cancer, intrinsic apoptosis, $\mathrm{G} 2 / \mathrm{M}$ phase arrest use $(4,5)$. Therefore, identification of additional therapeutic modalities in gastric cancer will be helpful in promoting the feasibility of therapeutic regimens in the clinical setting.

Over the past decades, a variety of natural components have been investigated for their anticancer activity, and many of them have exhibited a promising potential for cancer treatment (6-8). Tanshinone is isolated from the roots of Salvia miltiorrhiza ('tanshen'), a well-known traditional Chinese medicine, and possesses a diverse pharmacological profile (9). Tanshinone has been widely used in the treatment of coronary heart diseases, particularly angina pectoris and myocardial infarction, of inflammatory diseases, including chronic hepatitis, arthritis, and endangitis. In addition, it has antioxidant activity and liver protective effects $(10,11)$. The pharmacological features of Tanshinone IIA (Tan IIA, $\mathrm{C}_{19} \mathrm{H}_{18} \mathrm{O}_{3}$; Fig. 1), a major constituent of Salvia miltiorrhiza, have been widely studied. Interestingly, in addition to its biological activity, recent findings indicate that Tan IIA also exhibits anticancer activity in many cancer cells including leukemia, liver cancer, breast cancer, and gliomas (12-15). Based on its activity in other cancer cells, we speculated that Tan IIA may be a potential candidate for the treatment of gastric cancer. The anticancer effects of Tan IIA in gastric cancer have not been extensively studied. Therefore, in the present study, we provide a comprehensive investigation of the cytotoxicity of Tan IIA in gastric cancer cells in vitro and in a mouse xenograft model.

\section{Materials and methods}

Chemicals and reagents. Tan IIA and anti-human $\beta$-actin were purchased from Santa Cruz Biotechnology (Santa Cruz, CA). Anti-human cleaved poly (ADP-ribose) polymerase (PARP), anti-human cleaved caspase3, cytochrome c (cyto-c), and all secondary antibodies were purchased from Cell Signaling Technology (Danvers, MA). Anti-human Bcl-2, Bax, and purified mouse anti-human caspase 9 were purchased from BD Biosciences (Franklin Lakes, NJ).

Cell culture. The gastric cancer cell lines MKN45 and SGC7901 were generously provided by the Shanghai Cancer Institute. MKN45 and SGC7901 cells were grown as adherent monolayers in flasks in RPMI-1640 with $10 \%$ fetal bovine serum (FBS), at $37^{\circ} \mathrm{C}$ in a humidified atmosphere of $5 \% \mathrm{CO}_{2}$. 
Analysis of cell growth inhibition. Cell growth inhibition was determined colorimetrically using the 3-(4,5-dimethylthiazol2-yl)-2,5-diphenyltetrazolium bromide (MTT) reagent. Initially, $5 \times 10^{3}$ cells/well were seeded in 96 -well plates for $24 \mathrm{~h}$ before exposure to drug treatment, and then $10 \mu$ l MTT solution $(5 \mathrm{mg} / \mathrm{ml}$ in PBS) was added to each well for an additional $3 \mathrm{~h}$ at $37^{\circ} \mathrm{C}$. The medium was replaced with $200 \mu \mathrm{l}$ dimethyl sulfoxide (DMSO) for $1 \mathrm{~h}$ and the absorbance was determined at $570 \mathrm{~nm}$ and recorded with a microplate reader. Cells without Tan IIA treatment served as control.

Analysis of apoptotic cell population and cell cycle distribution for gastric cancer cells using a flow cytometry assay. Cell apoptosis quantification and cell cycle distribution was determined by flow cytometry analysis. Briefly, $3 \times 10^{5}$ cells were seeded in 6-well plates and exposed to various concentrations of Tan IIA treatments. For apoptosis quantification, the floating and trypsinized adherent cells were collected and prepared for detection according to the manufacturer's instructions enclosed in the FITC- Annexin V Apoptosis Detection Kit (BD Biosciences). For cell cycle distribution, adherent cells after drug treatment were collected and fixed with $75 \%$ ethanol $(\mathrm{v} / \mathrm{v})$, stained with propidium iodide (PI) and analyzed by a flow cytometer.

Western blot analysis. RIPA buffer in the presence of protease inhibitor cocktail (Roche) was used to extract total protein. The lysate was centrifuged at $12,000 \mathrm{rpm}$ at $4^{\circ} \mathrm{C}$ for $10 \mathrm{~min}$ to remove insoluble material. Cytosolic protein without mitochondrial protein was extracted using the Proteo Extract Cytosol/Mitochondria Fractionation kit (Calbiochem) acording to the manufacturer's instructions. The protein content was determined using a DC protein assay kit (Bio-Rad). The supernatant (30 $\mu \mathrm{g}$ of protein) was subjected to $8-15 \%$ SDS-PAGE electrophoresis. Proteins were electroblotted onto nitrocellulose membranes. After blocking with 5\% nonfat milk for $1 \mathrm{~h}$, the blots were probed with primary antibodies overnight at $4^{\circ} \mathrm{C}$. The blots were then incubated with HRP-conjugated antiIgG for $2 \mathrm{~h}$. After washing, the blots were detected using an enhanced chemiluminescence reagent (Amersham Pharmacia Biotech).

Subcutaneous tumor model of gastric cancer. Four-week-old male nude mice were purchased from the Shanghai Slac Animal Center (Shanghai, China). Aliquots of cells $\left(1 \times 10^{7}\right.$ cells $\left./ 100 \mu \mathrm{l}\right)$ were injected subcutaneously (s.c.) into the right inguinal area of the mice. When tumor diameter reached $\sim 5 \mathrm{~mm}$, tumorbearing mice were randomized into 3 experimental groups $(10 \mathrm{mg} / \mathrm{kg}$ Tan IIA group, $30 \mathrm{mg} / \mathrm{kg}$ Tan IIA group and the control group with the same volume of saline). Tan IIA was dissolved in $0.5 \%$ carboxymethyl cellulose (CMC). Treatments were administered via s.c. injection 5 times/week for 3 weeks. The tumor growth and body weight of the mice were monitored twice per week. Mice were weighed, and tumor volumes were assessed by measuring the 2 perpendicular dimensions using a caliper and the formula $(\operatorname{axb} 2) / 2$, where $\mathrm{a}$ is the larger and $b$ is the smaller dimension of the tumor. When treatment was finished, the mice were sacrificed and the tumors were excised. Tumor tissues were trimmed of extraneous fat or connective tissue, and homogenized in RIPA buffer (100 mg

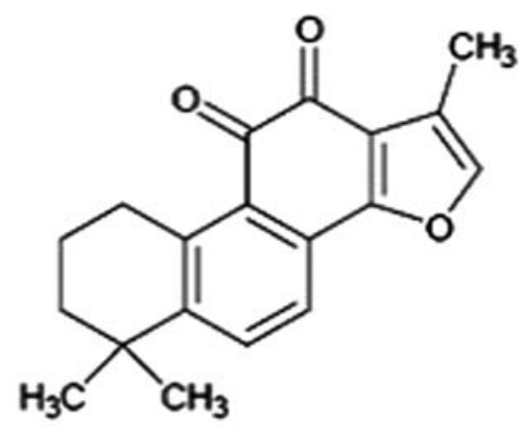

Figure 1. Chemical structure of Tanshinone IIA (Tan IIA).

tumor tissue/1 ml RIPA) and prepared for Western blotting analysis.

Statistical analysis. Statistical analysis was performed using the unpaired Student's t-test and an analysis of variance (oneway ANOVA). The accepted level of significance was P-value $<0.05$.

\section{Results}

Tan IIA exhibits antiproliferative activity in gastric cancer cells. To evaluate the inhibitory effect of Tan IIA on gastric cancer cells, we exposed MKN45 and SGC7901 cells to different concentrations of Tan IIA for $24 \mathrm{~h}$ and $48 \mathrm{~h}$. As shown in Fig. 2, the relative viability of cells subsequent to Tan IIA treatment proved to be both concentration- and time-dependent $(\mathrm{P}<0.05)$. The $\mathrm{IC}_{50}$ of Tan IIA at $48 \mathrm{~h}$ was $8.35 \mu \mathrm{g} / \mathrm{ml}$ in MKN45 cells and $10.78 \mu \mathrm{g} / \mathrm{ml}$ in SGC7901 cells respectively.

Apoptotic effect induced by Tanshinone IIA in gastric cancer cells. In addition to the antiproliferative activity induced by Tan IIA, we also investigated apoptosis exerted by Tan IIA in gastric cancer cells. Apoptosis of MKN45 cells and SGC7901 cells induced by Tan IIA was analyzed by FACS flow cytometry. As shown in Fig. 3A, the apoptotic index was directly related to both drug concentration and exposure time in gastric cancer cells. In MKN45 cells, the proportion of apoptotic cells treated by $6 \mu \mathrm{g} / \mathrm{ml}$ and $10 \mu \mathrm{g} / \mathrm{ml}$ Tan IIA for $24 \mathrm{~h}$ was $13.1 \pm 1.7 \%$ and $27.2 \pm 1.1 \%$ respectively, and treatment with $10 \mu \mathrm{g} / \mathrm{ml}$ Tan IIA for 24 and $48 \mathrm{~h}$ induced apoptotic fractions of $27.2 \pm 1.1 \%$ and $54.2 \pm 3.8 \%$ respectively (Fig. 3B). In SGC7901 cells, the proportion of apoptotic cells treated by 6 and $10 \mu \mathrm{g} / \mathrm{ml}$ Tan IIA for $24 \mathrm{~h}$ was $9.3 \pm 1.5 \%$ and $16.6 \pm 0.7 \%$ respectively, and treatment with $10 \mu \mathrm{g} / \mathrm{ml}$ Tan IIA for 24 and $48 \mathrm{~h}$ induced apoptotic fractions of $16.6 \pm 0.7 \%$ and $45.8 \pm 2.2 \%$ respectively (Fig. 3B).

We then examined the apoptotic effect of Tan IIA on MKN45 cells and SGC7901 cells through detecting the protein expression levels of the apoptosis-related proteins, the executor caspase 3 and its target, PARP. As shown in Fig. 3C, with increasing concentrations of Tan IIA, the pro-caspase3 and prototype of PARP expression levels were downregulated, and they were both cleaved to their active forms, cleaved-caspase 3 and cleaved-PARP in both the MKN45 and the SGC7901 cells. 
MKN45

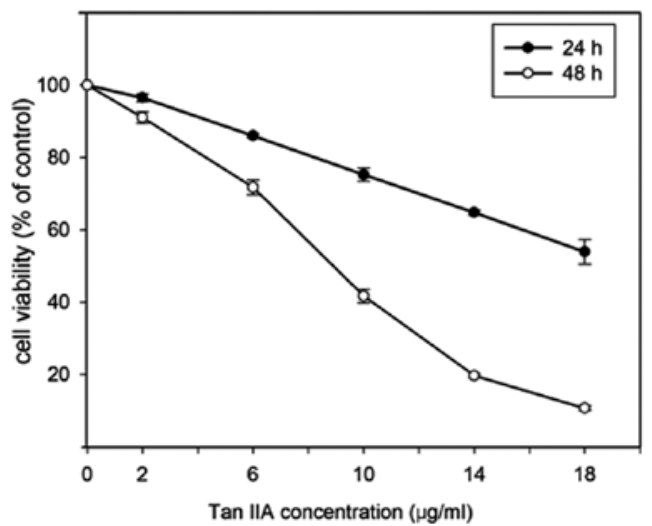

SGC7901

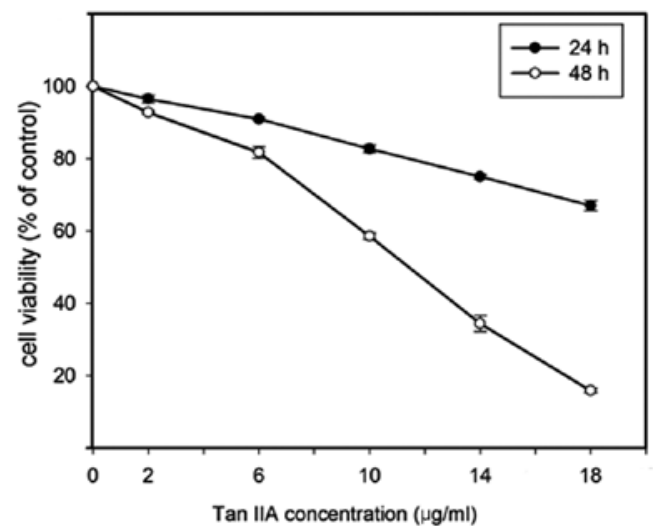

Figure 2. Tan IIA inhibits the growth of gastric cancer cells in a time and concentration-dependent manner. MKN45 cells and SGC7901 cells were treated with increasing concentrations of 2,6,10,14 and $18 \mu \mathrm{g} / \mathrm{ml}$ Tan IIA for 24 and $48 \mathrm{~h}$ and cell viability was determined by the MTT assay. Data are representative of values from at least three independent experiments.

A

$24 \mathrm{~h}$
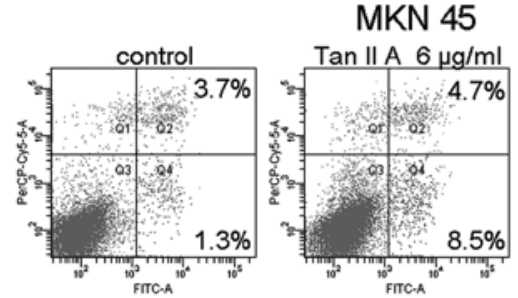

MKN 45
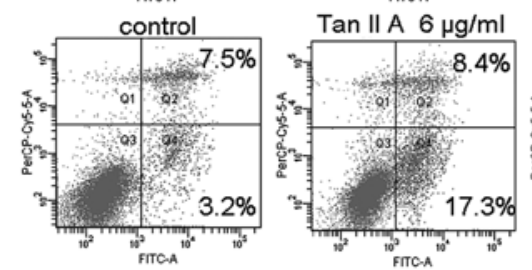
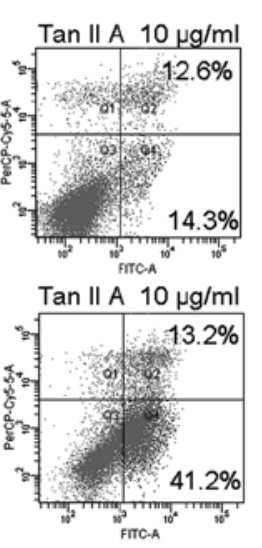

B
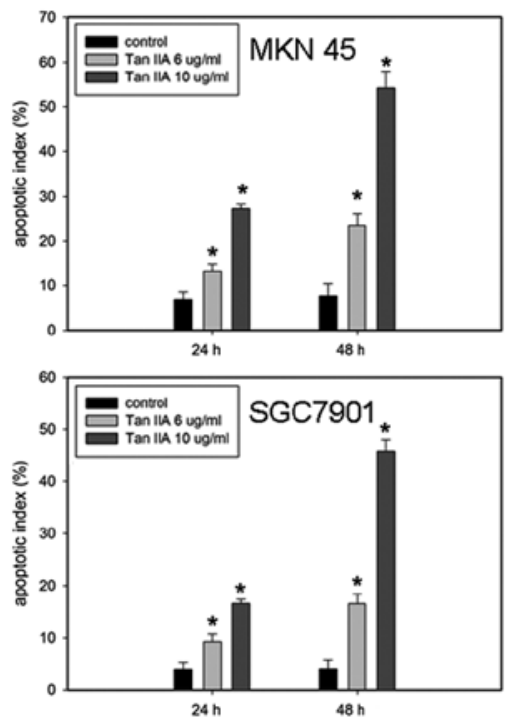

C
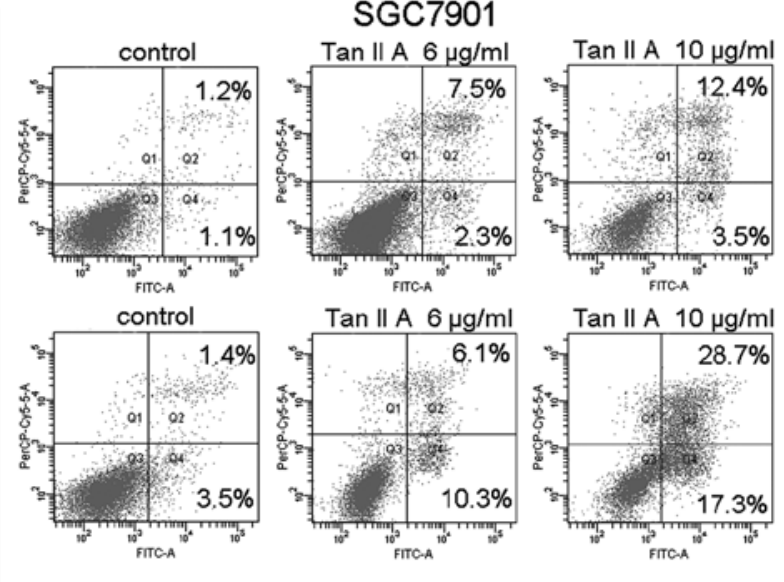

MKN 45

SGC7901

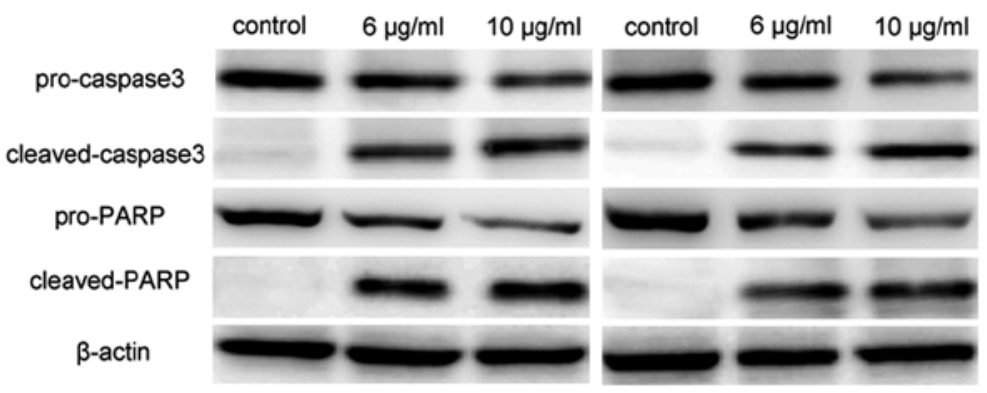

Figure 3. Tan IIA induces apoptosis in gastric cancer cells. (A) Representative dot-plots illustrating the apoptotic status in MKN45 and SGC7901 cells. Flow cytometric detection of apoptosis via Annexin V-FITC/PI staining in both cells treated with 6 and $10 \mu \mathrm{g} / \mathrm{ml}$ Tan IIA for 24 and $48 \mathrm{~h}$. (B) Data summary and analysis of apoptotic index in MKN45 and SGC7901 cells. Data are representative of values from at least three independent experiments. The percentage of cellular apoptosis is represented by Q2+Q4. * $\mathrm{P}<0.05$ vs. control. (C) Western blot analysis of apoptosis-related factors in gastric cancer cells. MKN45 and SGC7901 cells were treated with 6 and $10 \mu \mathrm{g} / \mathrm{ml}$ Tan IIA for $48 \mathrm{~h}$.

Tan IIA causes cell cycle arrest in the G2/M phase and triggers the intrinsic apoptosis pathway. In order to investigate the underlying mechanism of growth inhibition induced by
Tan IIA, we detected the cell cycle distribution state of MKN45 and SGC7901 cells exposed to $10 \mu \mathrm{g} / \mathrm{ml}$ of Tan IIA for 24 or $48 \mathrm{~h}$. As shown in Table I, compared to non-treated cells, 
Table I. Cell cycle distribution of MKN45 and SGC7901 cells after treatment with $10 \mu \mathrm{g} / \mathrm{ml}$ Tan IIA.

\begin{tabular}{lcccc}
\hline Group & Time $(\mathrm{h})$ & G0/G1 $(\%)$ & S $(\%)$ & G2/M $(\%)$ \\
\hline MKN45 cells & & & & \\
Control & 24 & $41.4 \pm 1.2$ & $37.3 \pm 1.4$ & $22.3 \pm 2.0$ \\
& 48 & $40.5 \pm 0.8$ & $35.7 \pm 2.1$ & $24.8 \pm 1.4$ \\
Tan IIA & 24 & $36.7 \pm 2.3$ & $34.5 \pm 1.1$ & $29.8 \pm 1.5^{\mathrm{a}}$ \\
& 48 & $20.2 \pm 0.4$ & & \\
SGC7901 cells & & & $47.7 \pm 2.4$ & \\
Control & 24 & $33.5 \pm 1.5$ & $48.4 \pm 2.1$ & \\
& 48 & $31.7 \pm 1.4$ & $45.1 \pm 2.3$ & \\
Tan IIA & 24 & $30.7 \pm 2.1$ & $29.7 \pm 3.4$ & \\
\end{tabular}

${ }^{\text {aP }}<0.05$ compared to the control group $(n=3)$

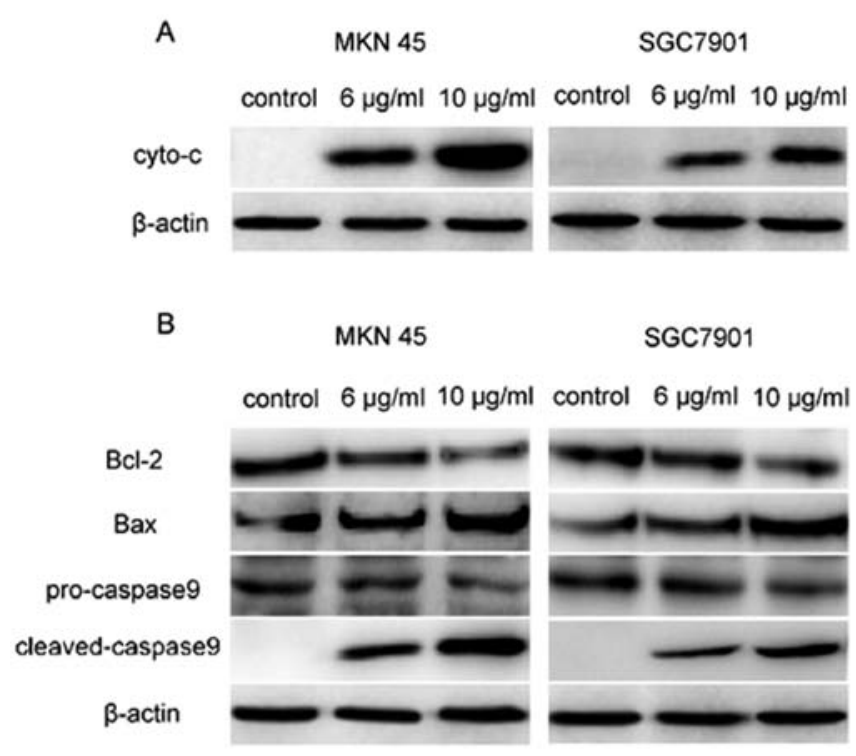

Figure 4. Exposure of gastric cancer cells to Tan IIA triggered the intrinsic apoptosis pathway. (A) Western blot analysis of cytosolic cytochrome c (cyto-c) in gastric cancer cells. MKN45 and SGC7901 cells were treated with 6 or $10 \mu \mathrm{g} / \mathrm{ml}$ Tan IIA for $24 \mathrm{~h}$. (B) Western blot analysis of molecular markers involved in mitochondria-related apoptosis. MKN45 and SGC7901 cells were treated with 6 or $10 \mu \mathrm{g} / \mathrm{ml}$ Tan IIA for $48 \mathrm{~h}$.

Tan IIA caused a significant accumulation of MKN45 cells in the G2/M phase, while decreased the population of cells in the G1 and S phase, and this effect was time-dependent. The redistribution of the cell cycle caused by Tan IIA also occurred in SGC7901 cells.

We then investigated the mechanism responsible for the Tan IIA-induced apoptosis. When MKN45 and SGC7901 cells were treated by increasing concentrations of Tan IIA for $24 \mathrm{~h}$, the mitochondria-related apoptotic pathway was switched on. Cytochrome c (cyto-c) leakage from the mitochondrial to the cytosolic fraction is used to monitor the mitochondrionrelated caspase cascade. As shown in Fig. 4A, the cytosolic cyto-c protein expression levels in MKN45 and SGC7901 cells treated with $6 \mu \mathrm{g} / \mathrm{ml} 10 \mu \mathrm{g} / \mathrm{ml}$ of Tan IIA for $24 \mathrm{~h}$ was signifi- cantly up-regulated compared with that of the control group. In addition, the expression patterns of proteins involved in the mitochondria-related apoptosis pathway were also examined (Fig. 4B). Tan IIA treatment resulted in a down-regulation of Bcl-2 and in up-regulation of Bax protein expression. The expression of pro-caspase 9 was decreased and its cleaved form was up-regulated when exposed to Tan IIA.

Tan IIA inhibits subcutaneous tumor growth in the mouse xenograft model of gastric cancer. To further confirm the anticancer effect of Tan IIA on gastric cancer, an in vivo experiment was carried out using a subcutaneous mouse xenograft model with MKN45 cells and SGC7901 cells as described in Materials and methods. Tumor growth was significantly inhibited in both tumor models (Fig. 5). We found that tumor growth suppression in the MKN45 cell model was 26 and $72 \%$ compared to that of the control group when treated with 10 and $30 \mathrm{mg} / \mathrm{kg}$ Tan IIA for 3 weeks, respectively $(\mathrm{P}<0.05$; Fig. 5A, left panel). Tumor growth suppression with SGC7901 cells was not significant in the $10 \mathrm{mg} / \mathrm{kg}$ Tan IIA group. However, when given a treatment of $30 \mathrm{mg} / \mathrm{kg}$ Tan IIA for 3 weeks, tumor volume in the subcutaneous mouse xenograft model with SGC7901 cells was decreased by $65 \%$ as compared to that of the control group ( $\mathrm{P}<0.05$; Fig. 5A, right panel).

At the end of the experiment, mice were sacrificed and the tumors were excised from the body. The average tumor weights in the Tan IIA $10 \mathrm{mg} / \mathrm{kg}$ and $30 \mathrm{mg} / \mathrm{kg}$-treated groups of MKN45 cells were $1.23 \pm 0.11 \mathrm{~g}$ and $0.54 \pm 0.08 \mathrm{~g}$, significantly lower compared to the $1.58 \pm 0.15 \mathrm{~g}$ tumor weight average in the control group $(\mathrm{P}<0.05$; Fig. $5 \mathrm{~B}$, left panel). The average tumor weight in the Tan IIA $30 \mathrm{mg} / \mathrm{kg}$-treated group of SGC7901 cells decreased to $0.78 \pm 0.07 \mathrm{~g}$ as compared to $2.31 \pm 0.21 \mathrm{~g}$ in the control group, $\mathrm{P}<0.05$ (Fig. 5B, right panel).

Tan IIA induces apoptosis of gastric cancer cells in vivo. In order to validate the mechanism by which Tan IIA exerts its antitumor effect, we investigated the in vivo expression of some of the key apoptosis-related proteins examined in the in vitro assay. The expression trend of Bcl-2, Bax and pro-caspase9 were all in accord with the in vitro studies (Fig. 6). 

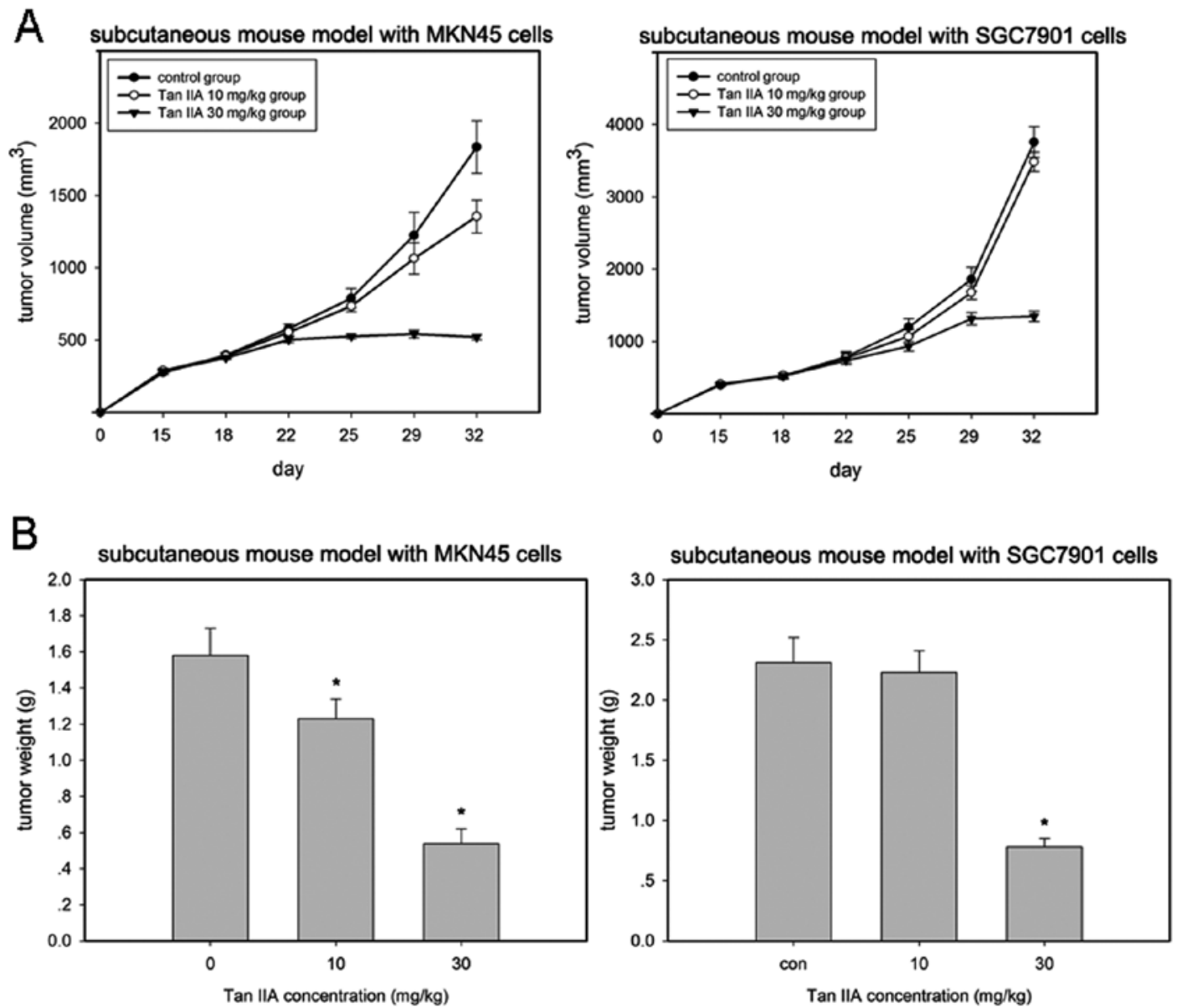

Figure 5. Tan IIA antitumor activity in nude mice bearing human gastric cancer cells. (A) Tumor growth curve for mice with subcutaneous tumors treated with Tan IIA. The mice (6/group) bearing tumors received daily s.c. injections of 10 or $30 \mathrm{mg} / \mathrm{kg} / \mathrm{day}$ Tan IIA 5 times/week for 3 weeks (the control group mice received an equal volume of saline). Data represent the mean tumor size for each group. (B) Tumor tissue was excised from the mice and its weight was measured. Data represent the mean tumor weight for each group. ${ }^{*} \mathrm{P}<0.05$ vs. the control group.

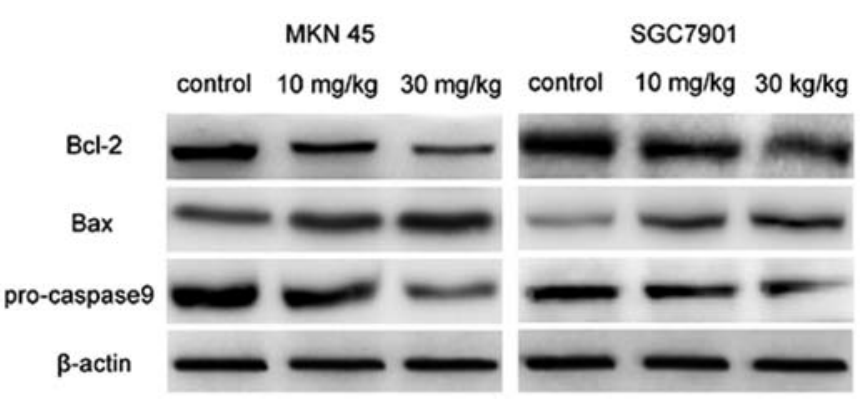

Figure 6. Western blot analysis of related markers of the intrinsic apoptosis pathway on excised tumors.

\section{Discussion}

As a phytochemical derived from the roots of Salvia miltiorrhiza Bunge, Tan IIA has been reported to have anti-inflammatory and antioxidant activities. Evidence is accumulating that Tan IIA may exhibit a promising anticancer activity (10). In the present study, we found that Tan IIA can significantly induce gastric cancer cell growth inhibition and apoptosis through cell cycle arrest and the mitochondria-dependent apoptotic signaling pathway in a time- and concentration-dependent manner. The antiproliferative activity of Tan IIA in gastric cancer was shown partly via cell cycle arrest in G2/M phase. As mentioned in previous studies, Tan IIA has been indicated to exert cytotoxicity to cancer cells through inducing cell cycle arrest in the G2/M phase in prostate cancer cells and HeLa cells $(16,17)$. In addition, a study on the effect of Tan IIA on gastric cancer cells indicated that Tan IIA can arrest MKN45 cells in the G2/M phase which led to an obvious accumulation of G2/M phase cells, while it decreased the number of G0/G1 phase cells, which was in accord with our results (18). However, the effect of Tan IIA on the cell cycle may not always be the same. One predominant event in liver fibrosis is the activation of hepatic stellate cells (HSCs). The selective clearance of activated HSCs is the therapeutic goal in anti-liver fibrosis (19). The study of the effect of Tan IIA on HSCs indicated that Tan IIA can exert a significant anti-fibrotic activity through inhibiting HSCs viability and arresting the cell cycle in the $S$ phase (20). We speculate that the differences in the cell cycle distribution may be related with the cells types, and also indicate that Tan IIA possesses a complex pharmacology mechanism against tumor cells or non-tumor cells.

Apoptosis, also named programmed cell death, is a very important physical phenomenon during development and injury repair. In addition, as a critical mechanism, most anticancer drugs exert their cytotoxic effect through 
inducing cells apoptosis. In general, apoptosis can be exerted through two different pathways, the mitochondria-dependent intrinsic-apoptotic pathway, and the death receptor-mediated extrinsic-apoptotic pathway. In our study, we found that Tan IIA activated the release of cyto-c into the cytoplasm, and increased the $\mathrm{Bax} / \mathrm{Bcl}-2$ ratio and the cleavage of the prototype of caspase9, events which all contribute to the intrinsic apoptotic signaling pathway. It has been indicated that the intrinsic death signaling pathway is the preferred mechanism employed by most anticancer compounds, and Bcl-2 family members play an important role during this process (21). Interestingly, more and more evidence shows that in addition to a conventional role in regulating mitochondrial outer membrane permeabilization, Bcl-2 also possesses another function in regulating the cellular redox state $(22,23)$. Previous studies have indicated that overexpression of $\mathrm{Bcl}-2$ can cause an increase in mitochondrial metabolism, which induced a slight pro-oxidant state that favors cancer cells in a survival advantage $(22,24)$. We also noticed that Tan IIA is an efficient antioxidant, and its role in modulating the expression of $\mathrm{Bcl}-2$ may contribute to the dysregulation of the mitochondrial metabolism and to the imbalance of the cellular redox state, events which contribute to its apoptotic effect.

In conclusion, evidence present in our study indicates that Tan IIA has a potent antitumor effect in vitro and in a gastric cancer mouse xenograft model, suggesting that Tan IIA may be a promising herbal medicine candidate in the clinical treatment of gastric cancer.

\section{Acknowledgements}

This work was supported by grants from Shanghai Pujiang Program (08PJ14018), and the National Natural Science Foundation of China (30970684).

\section{References}

1. Saif MW, Makrilia N, Zalonis A, Merikas M and Syrigos K: Gastric cancer in the elderly: an overview. Eur J Surg Oncol 36: 709-717, 2010.

2. Bittoni A, Maccaroni E, Scartozzi M, Berardi R and Cascinu S: Chemotherapy for locally advanced and metastatic gastric cancer: state of the art and future perspectives. Eur Rev Med Pharmacol Sci 14: 309-314, 2010.

3. Roukos DH: Current status and future perspectives in gastric cancer management. Cancer Treat Rev 26: 243-255, 2000.

4. Groth S, Nielsen H, Sorensen JB, Christensen AB, Pedersen AG and Rorth M: Acute and long-term nephrotoxicity of cis-platinum in man. Cancer Chemother Pharmacol 17: 191-196, 1986.

5. Stewart DJ: Mechanisms of resistance to cisplatin and carboplatin. Crit Rev Oncol Hematol 63: 12-31, 2007.

6. Efferth T, Dunstan H, Sauerbrey A, Miyachi H and Chitambar CR: The anti-malarial artesunate is also active against cancer. Int $\mathrm{J}$ Oncol 18: 767-773, 2001.
7. Gutheil WG, Reed G, Ray A and Dhar A: Crocetin: an agent derived from saffron for prevention and therapy for cancer. Curr Pharm Biotechnol: April 5, 2011 (Epub ahead of print).

8. Gao H, Lamusta J, Zhang WF, Salmonsen R, Liu Y, O'Connell E, Evans JE, Burstein S and Chen JJ: Tumor cell selective cytotoxicity and apoptosis induction by an herbal preparation from Brucea javanica. N A J Med Sci 4: 62-66, 2011.

9. Chiu TL and Su CC: Tanshinone IIA induces apoptosis in human lung cancer A549 cells through the induction of reactive oxygen species and decreasing the mitochondrial membrane potential. Int J Mol Med 25: 231-236, 2010.

10. Dong Y, Morris-Natschke SL and Lee KH: Biosynthesis, total syntheses, and antitumor activity of tanshinones and their analogs as potential therapeutic agents. Nat Prod Rep 28: 529-542, 2011.

11. Wang X, Morris-Natschke SL and Lee KH: New developments in the chemistry and biology of the bioactive constituents of Tanshen. Med Res Rev 27: 133-148, 2007.

12. Yoon Y,Kim YO, Jeon WK, Park HJ and Sung HJ: Tanshinone IIA isolated from Salvia miltiorrhiza BUNGE induced apoptosis in HL60 human premyelocy tic leukemia cell line. J Ethnopharmacol 68: 121-127, 1999.

13. Wang X, Wei Y, Yuan S, Liu G, Lu Y, Zhang J and Wang W: Potential anticancer activity of tanshinone IIA against human breast cancer. Int J Cancer 116: 799-807, 2005.

14. Yuxian X, Feng T, Ren L and Zhengcai L: Tanshinone II-A inhibits invasion and metastasis of human hepatocellular carcinoma cells in vitro and in vivo. Tumori 95: 789-795, 2009.

15. Tang C, Xue HL, Huang HB and Wang XG: Tanshinone IIA inhibits constitutive STAT3 activation, suppresses proliferation, and induces apoptosis in rat C6 glioma cells. Neurosci Lett 470: 126-129, 2010.

16. Gong Y, Li Y, Lu Y, Li L, Abdolmaleky H, Blackburn GL and Zhou JR: Bioactive tanshinones in Salvia miltiorrhiza inhibit the growth of prostate cancer cells in vitro and in mice. Int J Cancer 129: 1042-1052, 2011.

17. Pan TL, Hung YC, Wang PW, Chen ST, Hsu TK, Sintupisut N, Cheng CS and Lyu PC: Functional proteomic and structural insights into molecular targets related to the growth inhibitory effect of tanshinone IIA on HeLa cells. Proteomics 10: 914-929, 2010.

18. Dong X, Dong J and Peng G: Growth-inhibiting and apoptosisinducing effects of Tanshinone II A on human gastric carcinoma cells. J Huazhong Univ Sci Technolog Med Sci 27: 706-709, 2007.

19. Li JT, Liao ZX, Ping J, Xu D and Wang H: Molecular mechanism of hepatic stellate cell activation and antifibrotic therapeutic strategies. J Gastroenterol 43: 419-428, 2008.

20. Che XH, Park EJ, Zhao YZ, Kim WH and Sohn DH: Tanshinone IIA induces apoptosis and $S$ phase cell cycle arrest in activated rat hepatic stellate cells. Basic Clin Pharmacol Toxicol 106: 30-37, 2010.

21. Low IC, Kang J, and Pervaiz S: Bcl-2: a prime regulator of mitochondrial redox metabolism in cancer cells. Antioxid Redox Signal: July 12, 2011 (Epub ahead of print).

22. Chen ZX and Pervaiz S: Bcl-2 induces pro-oxidant state by engaging mitochondrial respiration in tumor cells. Cell Death Differ 14: 617-1627, 2007.

23. Chen ZX and Pervaiz S: Involvement of cytochrome c oxidase subunits $\mathrm{Va}$ and $\mathrm{Vb}$ in the regulation of cancer cell metabolism by Bcl-2. Cell Death Differ 17: 408-420, 2010.

24. Krishna S, Low IC and Pervaiz S: Regulation of mitochondrial metabolism: yet another facet in the biology of the oncoprotein Bcl-2. Biochem J 435: 545-551, 2011. 